\title{
DESEMPENHO EM CONSCIÊNCIA FONOLÓGICA, NOMEAÇÃO RÁPIDA, LEITURA E ESCRITA EM ESCOLARES COM DISLEXIA SECUNDÁRIA A RETARDO MENTAL E COM BOM DESEMPENHO ACADÊMICO
}

\section{Performance in phonological awareness, rapid naming, reading and writing in students with secondary dyslexia to mental retardation and with good academic performance}

Giseli Donadon Germano (1), Fábio Henrique Pinheiro (2), Niura Aparecida de Moura Ribeiro Padula ${ }^{(3)}$, Maria Dalva Lorencetti ${ }^{(4)}$, Simone Aparecida Capellini ${ }^{(5)}$

\begin{abstract}
RESUMO
Objetivos: caracterizar o desempenho em provas de consciência fonológica, de nomeação rápida e de leitura e escrita em escolares com dislexia secundária a retardo mental e com bom desempenho acadêmico. Método: participaram deste estudo 20 escolares da $1^{a}$ a $4^{a}$ séries do ensino fundamental da rede pública municipal da cidade de Marília-SP, com faixa etária de 8 a 12 anos de idade, de ambos os sexos, divididos em GI (10 escolares com o diagnóstico interdisciplinar de dislexia secundária a retardo mental leve) e GII (10 escolares com bom desempenho acadêmico, pareados segundo sexo e faixa etária com o Gl). Os escolares foram submetidos à Prova de Consciência Fonológica, à Prova de Leitura e Escrita e à Prova de Nomeação Automatizada Rápida. Resultados: houve diferença significante em relação ao desempenho dos escolares nas provas silábicas e fonêmicas, na prova de leitura e escrita e na prova de nomeção rápida; os escolares de GIl apresentaram melhor desempenho que os escolares de GI. Conclusão: houve relação entre os achados de velocidade de nomeação, leitura oral e escrita sob ditado, sendo relacionados ao déficit fonológico, à característica cognitivo-linguística do GI e à interferência da falta de instrução direta da correspondência grafofonêmica em situação de atividades acadêmicas para ambos os grupos.
\end{abstract}

DESCRITORES: Dislexia; Retardo Mental; Leitura; Avaliação

(1) Fonoaudióloga; Mestre e doutoranda do Programa de Pós-Graduação em Educação da Faculdade de Filosofia e Ciências da Universidade Estadual Paulista, FFC-UNESP, Marília-SP.

(2) Fonoaudiólogo; Mestre e doutorando do Programa de Pós-Graduação em Educação da Faculdade de Filosofia e Ciências da Universidade Estadual Paulista, FFC-UNESP, Marília-SP.

(3) Neurologista infantil; Docente do Departamento de Neurologia e Psiquiatria da Faculdade de Medicina de Botucatu da Universidade Estadual Paulista, UNESP, Botucatu-SP; Doutora em Ciências Médicas pela Faculdade de Ciências Médicas da Universidade Estadual de Campinas.

(4) Neuropsicóloga do Ambulatório de Desvios da Aprendizagem do Hospital das Clínicas da Faculdade de Medicina de Botucatu da Universidade Estadual Paulista, UNESP, Botucatu-SP.

\section{INTRODUÇÃO}

A dislexia do desenvolvimento é uma síndrome fenotipicamente hereditária, de origem genética, que consiste em uma pronunciada e persistente dificuldade na aquisição da leitura e nas habilidades de decodificação e soletração, resultantes de um déficit no componente fonológico da linguagem ${ }^{1,2}$.

(5) Fonoaudióloga; Docente do Departamento de Fonoaudiologia e do Programa de Pós-Graduação em Educação da Faculdade de Filosofia e Ciências da Universidade Estadual Paulista, FFC-UNESP, Marília-SP; Doutora em Ciências Médicas pela Faculdade de Ciências Médicas da Universidade Estadual de Campinas.

Conflito de interesses: inexistente 
O diagnóstico de dislexia implica na ausência de quaisquer transtornos de inteligência ou psiquiátricos. No entanto, é possível que uma criança com retardo mental leve receba o diagnóstico de dislexia, sendo esta considerada de origem secundária ${ }^{3}$. Assim, a dislexia passaria a ocorrer concomitantemente com outras condições, não sendo resultante diretamente dessas condições ${ }^{4}$.

Entre essas condições desfavoráveis encontrase o retardo mental, que é descrito, segundo o Manual Diagnóstico e Estatístico dos Distúrbios Mentais ${ }^{5}$, como um funcionamento mental significantemente inferior à média, acompanhado de limitações significantes no funcionamento adaptativo em pelo menos duas das seguintes áreas: comunicação, autocuidados, vida doméstica, habilidades sociais/interpessoais, uso de recursos comunitários, auto-suficiência, habilidades acadêmicas, trabalho, lazer, saúde, segurança.

A relação entre a habilidade fonológica e a aprendizagem da leitura vem sendo referida na literatura por décadas, sendo de alta significância para o escolar iniciante e relacionada às dificuldades de aprendizagem. Tal déficit tem se mostrado persistente com o passar do tempo, devido ao déficit na memória verbal e na rapidez de acesso da informação fonológica. A criança com dislexia apresenta dificuldade em representar mentalmente os padrões sonoros das palavras ${ }^{6-8}$.

A prova de nomeação rápida vem sendo descrita na literatura ${ }^{9}$ como a habilidade de processar símbolos visuais rapidamente, estando relacionada à aprendizagem da leitura e da escrita em ortografia alfabética, e a desordem nessa habilidade constitui um segundo déficit na consciência de fonemas. Também falham em testes que avaliam a memória de curta duração (memória de dígitos e de repetição de não palavras) e são lentos em testes de nomeação rápida, os quais avaliam a velocidade de acesso ao léxico familiar e a suas representações fonológicas ${ }^{8,10,11}$.

Estudos de comparação entre grupos de escolares com dislexia e com bom desempenho acadêmico tiveram como achados o desempenho inferior dos escolares com dislexia nas provas de nomeação rápida ${ }^{12}$, tarefas de leitura e escrita, aritmética $^{13}$ e em provas de consciência fonológica e habilidades auditivas ${ }^{14,15}$, evidenciando o déficit em acessar, processar e armazenar os códigos e as representações fonológicas.

A partir do exposto, este estudo teve por objetivos caracterizar o desempenho em provas de consciência fonológica, de nomeação rápida e de leitura e escrita em escolares com dislexia secundária a retardo mental e com bom desempenho acadêmico.

\section{MÉTODO}

Participaram deste estudo 20 escolares da $1^{\text {a }}$ a $4^{a}$ séries do ensino fundamental da rede pública municipal da cidade de Marília-SP, com faixa etária de 8 a 12 anos de idade, de ambos os sexos, divididos em:

- Grupo I (GI): composto por 10 escolares com o diagnóstico interdisciplinar de dislexia secundária ao retardo mental leve, atendidos no Centro de Estudos da Educação e Saúde - CEES/UNESP.

- Grupo II (GII): composto por 10 escolares com bom desempenho acadêmico de uma escola da rede municipal de ensino de Marília-SP, pareados segundo sexo e faixa etária com os sujeitos do grupo I (GI).

Os critérios de inclusão foram a assinatura do Termo de Consentimento Pós-Informado e, verificando-se o prontuário escolar, foram incluídos os alunos com laudo psicológico de atraso cognitivo leve que conseguiram realizar a leitura de pelo menos uma palavra inventada no momento da seleção dos escolares com atraso $\operatorname{cognitivo}^{16}$, garantindo, assim, a verificação de que os escolares do Gl eram capazes de realizar o mecanismo de conversão grafema-fonema no momento da leitura, o que caracteriza o domínio do princípio alfabético de leitura. Além disso, os escolares deveriam apresentar desempenho de 0,1 a 0,7 na Escala de Snellen ${ }^{17}$.

Foram excluídos deste estudo escolares com retardo mental de moderado a severo, escolares com o diagnóstico de distúrbio de aprendizagem e/ ou com alterações sensoriais.

Para a realização do presente estudo foram utilizados os seguintes procedimentos:

A) Termo de Consentimento Pós-Informado: Conforme resolução do Conselho Nacional de Saúde CNS 196/96, anteriormente ao início das avaliações, os pais ou responsáveis dos pacientes selecionados assinaram o Termo de Consentimento Pós-Informado para autorização da realização do estudo.

B) Prova de Consciência Fonológica ${ }^{18}$ : A Prova de Consciência Fonológica é composta de 10 subtestes, com 4 itens cada, que se referem a verificação das habilidades fonêmicas e silábicas (síntese, segmentação, manipulação e transposição) e supra-fonêmicas (rima e aliteração). O resultado desta prova é apresentado em forma de escore total.

C) Prova de Leitura e Escrita ${ }^{19}$ : O procedimento utilizado foi a leitura oral e a escrita sob ditado de 2 sublistas de 48 palavras reais (PR) e 48 
palavras inventadas (PIN), totalizando 96 palavras em cada categoria. As listas foram as mesmas para a tarefa de leitura oral e escrita sob ditado e foram analisadas de forma quantitativa, ou seja por freqüência de erros.

D) Prova de Nomeação Automatizada Rápida ${ }^{20}$ : Composta pelos subtestes de nomeação de cores, dígitos, letras e objetos. Os subtestes são compostos por 5 estímulos diferentes, os quais alternam-se entre si, formando ao todo 10 linhas seqüenciais, no total de 50 estímulos. O subteste de cores foi composto pelas cores: verde, vermelho, preto, azul e amarelo. O subteste de letras foi composto pelas letras $p, d$, $\mathrm{o}, \mathrm{a}, \mathrm{s}$. O subteste de dígito foi composto pelos seguintes números: 6, 2, 4, 9, 7 e o subteste de objetos: pente, guarda-chuvas, chave, relógio e tesoura. Os dados foram anotados em um protocolo específico para esse teste, constando os dados de tempo para cada subteste de caracterização dos erros cometidos.
Este trabalho foi aprovado pelo Comitê de Ética em Pesquisa (CEP) da Faculdade de Filosofia e Ciências da Universidade Estadual Paulista - Campus Marília, sob o número de protocolo 788/2003.

Os resultados foram analisados estatisticamente, utilizados o Teste T de Student e Análise de Variância, sendo que foi adotado o nível de significância de $5 \%(a=0,050)$. Para análise dos dados foi utilizado o programa SPSS (Statistical Package for Social Sciences), em sua versão 13.0.

\section{RESULTADOS}

A tabela 1 apresenta o desempenho dos escolares de Gl e Gll na Prova de Consciência Fonológica (PCF). Os resultados evidenciaram diferença significante para o desempenho dos escolares de GI e GII nos subtestes de síntese fonêmica, segmentação silábica e fonêmica, rima, aliteração, manipulação silábica e fonêmica, transposição silábica e, no escore total, evidenciando melhor desempenho dos escolares do Gll em relação ao GI.

Tabela 1 - Distribuição das médias desvio-padrão e significância $(p)$ do desempenho dos escolares dos GI e GII nos subtestes da Prova de Consciência Fonológica (PCF)

\begin{tabular}{|c|c|c|c|c|}
\hline Variável & Grupo & Média & Desvio-padrão & Valor de $p$ \\
\hline \multirow{2}{*}{ SiS } & 1 & 4,00 & 0,00 & \multirow{2}{*}{$>0,999$} \\
\hline & II & 4,00 & 0,00 & \\
\hline \multirow{2}{*}{ SiF } & $\mathrm{I}$ & 1,20 & 1,23 & \multirow{2}{*}{$0,017^{*}$} \\
\hline & II & 2,50 & 0,97 & \\
\hline \multirow{2}{*}{ SeS } & 1 & 1,80 & 1,69 & \multirow{2}{*}{$0,003^{*}$} \\
\hline & II & 3,90 & 0,32 & \\
\hline \multirow{2}{*}{ SeF } & 1 & 0,00 & 0,00 & \multirow{2}{*}{$0,012^{*}$} \\
\hline & II & 1,70 & 1,70 & \\
\hline \multirow{2}{*}{ RIM } & 1 & 1,10 & 1,29 & \multirow{2}{*}{$<0,001^{*}$} \\
\hline & II & 3,50 & 0,97 & \\
\hline \multirow{2}{*}{ ALI } & $\mathrm{I}$ & 0,60 & 1,26 & \multirow{2}{*}{$<0,001^{*}$} \\
\hline & II & 3,60 & 0,97 & \\
\hline \multirow{2}{*}{ Mans } & I & 1,50 & 1,51 & \multirow{2}{*}{$0,002^{*}$} \\
\hline & II & 3,60 & 0,84 & \\
\hline \multirow{2}{*}{ ManF } & 1 & 0,40 & 1,26 & \multirow{2}{*}{$0,003^{*}$} \\
\hline & II & 2,50 & 1,51 & \\
\hline \multirow{2}{*}{ Trans } & $\mathrm{I}$ & 0,60 & 1,26 & \multirow{2}{*}{$<0,001^{*}$} \\
\hline & II & 3,30 & 1,49 & \\
\hline \multirow{2}{*}{ TranF } & 1 & 0,00 & 0,00 & \multirow{2}{*}{0,081} \\
\hline & II & 0,90 & 1,45 & \\
\hline \multirow{2}{*}{ TOTAL } & $\mathrm{I}$ & 11,20 & 7,86 & \multirow{2}{*}{$<0,001^{*}$} \\
\hline & II & 29,50 & 6,87 & \\
\hline
\end{tabular}

Legenda: SiS: Síntese Silábica; SiF: Síntese Fonêmica; SeS: Segmentação Silábica; SeF: Segmentação Fonêmica; Rim: Rima; Alit: Aliteração; ManS: Manipulação Silábica; ManF: Manipulação Fonêmica; TrS: Transposição Silábica; TrF: Transposição Fonêmica; e Total. Teste T de Student, controlado pelo Teste de Levene para Igualdade de Variâncias. Valor de p: $<0,050$. 
Os resultados da Tabela 2 indicam a comparação do desempenho dos escolares de GI e GII quanto às provas silábicas, fonêmicas e suprafonêmicas da prova de consciência fonológica, sendo que houve diferença estatisticamente significante nos subtestes de síntese, segmentação e manipulação, evidenciando melhor desempenho desses escolares nas provas silábicas que fonêmicas. Em relação ao Gll, verificou-se, ainda, que houve diferença estatisticamente significante no subteste de transposição, evidenciando melhor desempenho desses escolares nas provas silábicas em relação às fonêmicas.

Tabela 2 - Distribuição das médias, desvio-padrão e significância (p) do desempenho dos escolares do GI de acordo com comparação intragrupo na prova de consciência fonológica

\begin{tabular}{|c|c|c|c|c|c|c|}
\hline \multirow[b]{2}{*}{ Variáveis } & \multicolumn{3}{|c|}{ GI } & \multicolumn{3}{|c|}{ GII } \\
\hline & Média & $\begin{array}{l}\text { Desvio- } \\
\text { padrão }\end{array}$ & Valor de $p$ & Média & $\begin{array}{l}\text { Desvio- } \\
\text { padrão }\end{array}$ & Valor de $p$ \\
\hline Sis & 4,00 & 0,00 & \multirow{2}{*}{$<0,001^{*}$} & 4,00 & 0,00 & \multirow{2}{*}{$0,001^{*}$} \\
\hline SiF & 1,20 & 1,23 & & 2,50 & 0,97 & \\
\hline Ses & 1,80 & 1,69 & \multirow{2}{*}{$0,008^{\star}$} & 3,90 & 0,32 & \multirow{2}{*}{$0,002^{*}$} \\
\hline SeF & 0,00 & 0,00 & & 1,70 & 1,70 & \\
\hline RIM & 1,10 & 1,29 & \multirow{2}{*}{0,096} & 3,50 & 0,97 & \multirow{2}{*}{0,343} \\
\hline ALI & 0,60 & 1,26 & & 3,60 & 0,97 & \\
\hline ManS & 1,50 & 1,51 & \multirow{2}{*}{$0,024^{*}$} & 3,60 & 0,84 & \multirow{2}{*}{$0,003^{*}$} \\
\hline ManF & 0,40 & 1,26 & & 2,50 & 1,51 & \\
\hline Trans & 0,60 & 1,26 & \multirow{2}{*}{0,168} & 3,30 & 1,49 & \multirow{2}{*}{$0,002^{*}$} \\
\hline TranF & 0,00 & 0,00 & & 0,90 & 1,45 & \\
\hline
\end{tabular}

Legenda: SiS: Síntese Silábica; SiF: Síntese Fonêmica; SeS: Segmentação Silábica; SeF: Segmentação Fonêmica; Rim: Rima; Alit: Aliteração; ManS: Manipulação Silábica; ManF: Manipulação Fonêmica; TrS: Transposição Silábica; TrF: Transposição Fonêmica; e Total. Teste T de Student para Dados Pareados. Valor de $\mathrm{p}:<0,050$.

A Tabela 3 indica os resultados dos escolares de Gl e Gll em relação à Prova de Leitura e Escrita. Em relação à Prova de Leitura Oral, observou-se diferença estatisticamente significante nas categorias de palavras referentes a palavras reais de baixa frequência regulares; palavras reais de alta frequência regulares; palavras reais de baixa frequência irregulares; palavras reais de alta frequência irregulares; palavras reais de alta frequência regra; palavras inventadas regulares; palavras inventadas regra, evidenciando que os escolares de $\mathrm{Gl}$ apresentaram maior média de erros em relação aos escolares de Gll. Em relação à Prova de Escrita sob Ditado, verificou-se diferença estatisticamente significante somente em relação à categoria de palavras reais de baixa frequencia regular, com maior média de erros dos escolares de Gl em relação aos escolares de GII.

A Tabela 4 indica os resultados Prova de Nomeação Rápida Automática (RAN), sendo verificado diferença estatisticamente significante para 0 desempenho dos escolares de GI e Gll apenas nos subtestes de letras e números, evidenciando melhor desempenho dos escolares do Gll em relação ao Gl nestes subtestes.

\section{DISCUSSÃO}

Neste estudo, evidenciou-se que apesar da diferença de média etária encontrada entre o Gl (10,4 anos de idade) e o Gll (9,7 anos de idade), os escolares estavam matriculados em sala de aula de ensino regular e na seriação adequada para o seu nível cognitivo-linguístico. Este fato aponta para a necessidade de um melhor conhecimento acerca do retardo mental limítrofe ou leve para oferecer condições adequadas de ensino e também compreender as chamadas "alterações da leitura e escrita" nessa população.

Entre os escolares que "não aprendem", encontram-se aqueles com retardo mental de grau leve e que muitas vezes não são percebidos 
Tabela 3 - Distribuição das médias de erros, desvio-padrão e significância ( $p$ ) do desempenho dos escolares de GI e Gll quanto à leitura de palavras reais e inventadas de baixa e alta frequência na Prova de Leitura Oral

\begin{tabular}{|c|c|c|c|c|c|c|c|}
\hline \multirow[b]{2}{*}{ Variável } & \multirow[b]{2}{*}{ Grupo } & \multicolumn{3}{|c|}{ Leitura } & \multicolumn{3}{|c|}{ Ditado } \\
\hline & & Média & $\begin{array}{l}\text { Desvio- } \\
\text { padrão }\end{array}$ & Valor de $p$ & Média & $\begin{array}{l}\text { Desvio- } \\
\text { padrão }\end{array}$ & Valor de $p$ \\
\hline \multirow{2}{*}{ PRBFR } & I & 4,00 & 3,06 & \multirow{2}{*}{$0,004^{*}$} & 4,20 & 3,61 & \multirow{2}{*}{$0,034^{*}$} \\
\hline & II & 0,30 & 0,68 & & 1,20 & 1,69 & \\
\hline \multirow{2}{*}{ PRAFR } & I & 5,80 & 5,63 & \multirow{2}{*}{$0,017^{*}$} & 2,30 & 2,36 & \multirow{2}{*}{0,202} \\
\hline & II & 0,60 & 1,27 & & 1,10 & 1,60 & \\
\hline \multirow{2}{*}{ PRBFI } & I & 3,80 & 3,12 & \multirow{2}{*}{$0,022^{*}$} & 4,70 & 3,37 & \multirow{2}{*}{0,763} \\
\hline & II & 1,00 & 1,25 & & 4,30 & 2,41 & \\
\hline \multirow{2}{*}{ PRAFI } & 1 & 3,60 & 3,84 & \multirow{2}{*}{$0,034^{*}$} & 2,20 & 2,86 & \multirow{2}{*}{0,816} \\
\hline & II & 0,50 & 1,27 & & 1,90 & 2,81 & \\
\hline \multirow{2}{*}{ PRBFRG } & 1 & 2,70 & 2,54 & \multirow{2}{*}{0,140} & 4,30 & 3,56 & \multirow{2}{*}{0,416} \\
\hline & II & 1,20 & 1,69 & & 3,20 & 2,15 & \\
\hline \multirow{2}{*}{ PRAFRG } & I & 5,90 & 4,86 & \multirow{2}{*}{$0,005^{\star}$} & 2,60 & 2,55 & \multirow{2}{*}{0,139} \\
\hline & II & 0,20 & 0,42 & & 1,10 & 1,66 & \\
\hline \multirow{2}{*}{ PIR } & I & 7,00 & 5,38 & \multirow{2}{*}{$0,030^{*}$} & 5,70 & 6,85 & \multirow{2}{*}{0,174} \\
\hline & II & 2,40 & 2,63 & & 2,40 & 2,07 & \\
\hline \multirow{2}{*}{ PII } & I & 7,30 & 5,95 & \multirow{2}{*}{0,066} & 7,40 & 7,83 & \multirow{2}{*}{0,155} \\
\hline & II & 3,10 & 2,89 & & 11,50 & 3,44 & \\
\hline \multirow{2}{*}{ PIRG } & I & 6,80 & 5,55 & \multirow{2}{*}{$0,037^{*}$} & 5,80 & 7,11 & \multirow{2}{*}{0,852} \\
\hline & II & 2,40 & 1,90 & & 5,30 & 4,30 & \\
\hline
\end{tabular}

Legenda: PRBFR: Palavras reais de baixa frequência regulares; PRAFR: Palavras reais de alta frequência regulares; PRBFI: Palavras reais de baixa frequência irregulares; PRAFI: Palavras reais de alta frequência irregulares; PRBFRG: Palavras reais de baixa frequência regra; PRAFRG: Palavras reais de alta frequência regra; PIR: Palavras inventadas regulares; PII: Palavras inventadas irregulares; PIRG: Palavras inventadas regra. Teste T de Student, controlado pelo Teste de Levene para Igualdade de Variâncias. Valor de $\mathrm{p}:<0,050$.

Tabela 4 - Distribuição das médias, desvio-padrão e significância (p) do desempenho dos escolares de GI e GIl nos subtestes no Teste de Nomeação Automática Rápida (RAN)

\begin{tabular}{lcccc}
\hline Variável & Grupo & Média & Desvio-padrão & Valor de $\mathbf{p}$ \\
\hline \multirow{2}{*}{ Cores } & I & 77,33 & 38,86 & \multirow{2}{*}{0,092} \\
& II & 44,33 & 7,91 & \multirow{2}{*}{$0,010^{*}$} \\
\hline \multirow{2}{*}{ Letras } & I & 59,33 & 18,08 & \multirow{2}{*}{$0,032^{*}$} \\
\hline \multirow{2}{*}{ Números } & II & 30,11 & 5,46 & \multirow{2}{*}{0,066} \\
\hline \multirow{2}{*}{ Objetos } & I & 56,67 & 23,23 & \\
& II & 28,89 & 5,93 & \\
\hline
\end{tabular}

Teste T de Student, controlado pelo Teste de Levene para Igualdade de Variâncias. Valor de p: $<0,050$. 
pelo professor e até mesmo confundidos com os chamados "alunos lentos ou atrasados". Essa rotulação, principalmente no início do período escolar, tem sido muito discutida. Ademais, sabe-se que seus efeitos levam ao entendimento de crianças que apresentam dificuldades de aprendizagem como incapazes, preguiçosas, lentas e perturbadas, contribuindo para o agravamento de suas dificuldades, reforçando a imagem negativa que fazem de si mesmas e não permitindo que sejam vistas como únicas ${ }^{21,22}$.

Neste estudo, em relação à Prova de Consciência Fonológica, verificou-se que os escolares de Gl apresentaram menor média de acerto na maioria das provas, tanto de habilidades silábicas quanto fonêmicas em relação aos escolares do GIl, com bom desempenho acadêmico, evidenciando o déficit fonológico de Gl, característica cognitivalinguística dos escolares com dislexia. Além disso, verificou-se também que os escolares de GI e Gll tiveram desempenho superior nas habilidades silábicas em relação às habilidades fonêmicas, evidenciando que ambos tiveram dificuldades em perceber estruturas fonêmicas, ou seja, o déficit em consciência fonêmica não é característica única de escolares que apresentam problemas de aprendizagem decorrentes de retardo mental ou não, e sim uma alteração que pode ser decorrente do ensino da base do sistema de escrita do português.

Desenvolver a consciência fonológica significa ter o conhecimento de manipular os segmentos. É a percepção de que as palavras faladas representam sequências de fonemas, os quais, se alterados, podem mudar o seu significado. Isto é, perceber que frases podem ser divididas em palavras e palavras podem ser divididas em sílabas, fonemas, rimas ou aliteração. A consciência fonológica refere-se à atenção que o indivíduo faz para acessar a construção sonora de uma linguagem oral e pode ser notada como, por exemplo, na realização de testes de deleção, adição ou subtração de sílabas/fonemas ${ }^{23-26}$.

Entretanto, em relação à prova de leitura, os resultados indicaram que os escolares com dislexia secundária ao retardo mental leve apresentaram maior média de erros em relação aos escolares com bom desempenho acadêmico, tanto para palavras reais quanto para palavras inventadas, evidenciando que estes escolares tiveram dificuldades na realização da conversão grafema-fonema. Em relação à prova de escrita sob ditado, verificou-se que os desempenhos dos escolares com dislexia secundária ao retardo mental leve e com bom desempenho acadêmico foram semelhantes, indicando a falta de instruções explícitas e adequadas sobre os aspectos da conversão fonema-grafema durante o processo de alfabetização para ambos os grupos.

A percepção da estrutura fonêmica da fala permite ao escolar utilizar um sistema gerativo para converter ortografia em fonologia, permitindo, assim, a leitura de qualquer palavra regular que envolva a correspondência grafofonêmica. A característica gerativa das ortografias alfabéticas permite a leitura de palavras novas e a aprendizagem por si mesmos, a partir da aplicação das regras de decodificação fonológica. O processo de decodificação fonológica contribui para que a criança forme a representação ortográfica da nova palavra, permitindo, dessa forma, que a palavra nova seja lida pela rota lexical. Portanto, é o processamento fonológico que permitirá à criança, posteriormente, realizar a leitura pela rota lexical|27,28.

Neste estudo, verificou-se que houve relação entre a consciência fonológica, a leitura e a escrita, sendo que vários autores ${ }^{29-31}$ referiram que a experiência da alfabetização representa papel fundamental na aquisição das habilidades fonêmicas, pois estão relacionadas à descoberta do princípio alfabético, enquanto que a rima não depende da alfabetização, pois se desenvolve naturalmente. Atividades como rima, aliteração, síntese e análise silábica surgem antes da alfabetização formal, enquanto outras habilidades, como segmentação e transposição de fonemas, são praticamente inexistentes no início da alfabetização. Ou seja, os escolares devem ter maior facilidade nas tarefas de síntese e análise silábica, passando para manipulação tanto silábica quanto fonêmica (tarefas intermediárias) até as tarefas fonêmicas.

Como consequência do exposto, os dados deste estudo apontaram a influência direta da relação entre as habilidades fonológica e de leitura, pois os escolares do Gll, com bom desempenho acadêmico apresentaram melhor desempenho em relação à leitura de palavras de alta frequência regular, irregular e regra do que de baixa frequência para essas categorias, enquanto que os escolares do GI tiveram maior dificuldade na leitura de palavras reais de baixa frequência e de palavras inventadas regulares, irregulares e regra. $\mathrm{O}$ fato de os escolares de Gl apresentarem maior dificuldade para realizar leitura de palavras reais e inventadas sugere a hipótese de que esses escolares possuem menor clareza quanto à percepção de estrutura fonológica das palavras quando as ouvem ou falam, o que sugere que estes escolares possuem melhor reconhecimento de palavras reais familiares pertencentes ao seu léxico de "inpuf" visual, favorecendo o reconhecimento global da palavra e apresentando dificuldade de analisar a palavra durante a leitura ou a escrita ${ }^{19,32}$. 
Outra informação importante deste estudo refere-se à nomeação automatizada rápida. A nomeação automatizada rápida tem sido considerada como uma habilidade da linguagem relacionada à consciência fonológica. Uma das razões dessa correlação entre nomeação automatizada rápida e consciência fonológica pode ser encontrada em alguns estudos ${ }^{8,33,34}$, que descreveram a nomeação automatizada rápida como um importante instrumento de verificação de atenção, velocidade de processamento, percepção, memória, léxico e processos articulatórios.

Estudos $^{11,} 35-38$ referiram que as habilidades de processamento fonológico, incluindo a nomeação automatizada rápida, são importantes e consistentes preditores da leitura e do ditado proficiente, e que geralmente a nomeação automatizada rápida reflete problemas de processamento fonológico em crianças com dificuldades de aprendizagem.

Os achados deste estudo evidenciaram que a velocidade de acesso ao léxico mental está diretamente relacionada com a habilidade de consciência fonológica, atenção e memória de trabalho, leitura e escrita, pois os escolares de Gl apresentaram alteração em tarefas de nomeação, evidenciando que os escolares de GI necessitaram de maior tempo para a nomeação de letras e números, sendo justificado pela literatura, já que a nomeação de estímulos com maior componentes fonológicos na palavra exige uso mais eficaz de processos atencionais e perceptuais visuais, como discriminação e análise-síntese, corroborando os achados da literatura $^{12,20,35,39}$.

Entretanto, verificou-se que o desempenho em nomeação foi semelhante entre o Gl e Gll para cores e objetos, evidenciando que a velocidade de nomeação sequencial de estímulos comuns, ou seja, a velocidade com que a criança verbaliza sequencialmente uma lista contendo símbolos básicos, não é diferente entre os escolares com retardo mental e os bons leitores. As relações estabelecidas neste estudo entre velocidade de nomeação e automatização dos estímulos apresentados com a capacidade de acesso lexical, discriminação visual, frequência de uso dos estímulos e competição para a apresentação no menor tempo possível para a nomeação dos códigos, são necessárias para o estabelecimento do mecanismo de conversão fonema-grafema exigido na realização da leitura e da escrita de um sistema de escrita com base alfabética, como o português.

Os dados deste estudo nos fazem refletir sobre a necessidade da atuação do fonoaudiológico na equipe interdisciplinar responsável pelo diagnóstico em aprendizagem, pois somente a partir do levantamento das manifestações cognitivo-linguísticas dos escolares com retardo mental leve é que será possível a definição do diagnóstico de dislexia do desenvolvimento de origem secundária, o que, de certa forma, contribuirá para a melhor adaptação desses escolares em situação de sala de aula, pois será possível estabelecer, juntamente com os professores, adaptações curriculares que proporcionem melhor qualidade de ensino para essa população.

\section{CONCLUSÃO}

A partir dos resultados deste estudo, concluiuse que houve relação entre habilidade fonológica, leitura e velocidade de nomeação. Os escolares de Gl apresentaram desempenho inferior aos escolares de Gll em relação às provas de consciência fonológica, com menor média de desempenho nas habilidades fonêmicas.

Os escolares de Gl apresentaram maior média de erros em relação a Gll na leitura de palavras reais e de pseudopalavras. Na prova de escrita sob ditado, os escolares de ambos os grupos apresentaram desempenho semelhantes para palavras reais e pseudopalavras. Na prova de nomeação rápida, os escolares de Gl apresentaram desempenho inferior aos escolares de Gll em relação às habilidade de nomeação de letras e números.

\section{AGRADECIMENTOS}

À Fundação de Amparo à Pesquisa do Estado de São Paulo - FAPESP (processo número 05/50277-2). 


\begin{abstract}
Purpose: to characterize the performance in phonological awareness, rapid naming, reading and writing in students with developmental dyslexia secondary to mental retardation and good readers. Method: 20 students from first to fourth grade of a public school of Marília - SP, both genders, from to 8 to 12-year old took part in this study, divided into Gl (10 students with interdisciplinary diagnosis of dyslexia, secondary to mild mental retardation) and GII (10 students with good academic development according to gender, age and grade level). The students were submitted to the Phonological Awareness Test, the Test of Reading and Writing and Rapid Automated Naming. Results: there were significant differences regarding the performance of students in the syllabic and phonemic tasks, reading and writing, Rapid Automated Naming, with better performance of the students of GII than GI. Conclusion: there was a relationship among the findings of naming speed, oral reading and writing under dictation, which were related with the phonological deficit, cognitive-linguistic characteristic of $\mathrm{Gl}$ and the interference and the lack of direct instruction of their spelling correspondence in a situation of academic activities for both groups.
\end{abstract}

KEYWORDS: Dyslexia; Mental Retardation; Reading; Evaluation

\section{REFERÊNCIAS}

1. Shaywitz S, Mody M, Shaywitz B. Neural mechanisms in dyslexia. Curr Dir Psychol Sci. 2006; 15 (6): 278- 81.

2. Penolazzi B, Spironelli C, Vio C, Angrilli A. Brain plasticity in developmental dyslexia after phonological treatment: A beta EEG band study. Behav Brain Res. 2010; 209: 179-82.

3. Galaburda A M, Cestnick L. Dislexia del desarrollo. Rev. Neurol. 2003; 36 (1 supl): s3-s9.

4. Hammill DD, Leigh JE, McNutt G, Larsen SC. A new definition of learning disabilities. Learn Disabil Q. 1981; 4: 336-42.

5. DSM IV - TR. Manual diagnóstico e estatístico de transtornos mentais. Edição Revisada. Porto Alegre: Artes Médicas, 2002.

6. Shaywitz S, Shaywitz B. Dyslexia (Specific Reading Disability). Biol Psychiatry. 2005; 57: 1301-9.

7. Beneventi B, Tønnessen FE, Ersland L, Hugdahl K. Executive working memory processes in dyslexia: Behavioral and fMR evidence. Scand J Psychol. 2010; 51: 192-202.

8. Van Der Lely HKJ, Marshall CR. Assessing Component Language Deficits in the Early Detection of Reading Difficulty Risk. J Learn Disabil. 2010; 43(4): 357-68.

9. Serrano F, Defior S. Dyslexia speed problems in a transparent orthography. Ann Dyslexia. 2008; 58(1): 85-95.

10. Desroches AS, Joanisse MF, Robertson EK. Specific phonological impairments in dyslexia revealed by eyetracking. Cognition. 2006; (100): B32-B42.

11. Hogan TP. A short report: word-level phonological and lexical characteristics and lexical characteristics interact to influence phoneme awareness. J Learn Disabil. 2010; 43(4): 346-56.

12. Capellini AS, Conrado TLBC. Desempenho de escolares com e sem dificuldades de aprendizagem de ensino particular em habilidade fonológica, nomeação rápida, leitura e escrita. Rev. CEFAC. 2009; 11 (2 supl): 183-93.

13. Capellini AS, Toyota P, Santos LCA, Lourencetti MD, Padula, NAMR. Caracterização do desempenho fonológico, da leitura e escrita em escolares com dislexia e distúrbio de aprendizagem. Cad Fil Psicol Educ. 2007; ano V(9): 37-70.

14. Capellini, S.A.; Germano, G.D.; Cardoso, A.C.V. Relação entre habilidades auditivas e fonológicas em crianças com dislexia do desenvolvimento: Habilidades auditiva-fonológica em disléxicos. Psicol Esc Educ. 2008; 12(1): 235-53.

15. Germano GD, Pinheiro FH, Capellini SA. Desempenho de escolares com dislexia do desenvolvimento em tarefas fonológicas e silábicas. Rev CEFAC. 2009; 11(2): 213-20.

16. Cardoso-Martins C, Frith U. Consciência fonológica e habilidade de leitura na Síndrome de Down. Psicol Reflex Crit. 1999; 12(1): 209-24.

17. Combos GM. Manual de emergências oftalmológicas. São Paulo: Manole, 1979.

18. Capovilla AGS, Capovilla FC. Prova de consciência fonológica: desenvolvimento de dez habilidades da pré-escola à segunda série. Temas Desenvolv. 1998; 7(37): 14-20. 
19. Pinheiro AMV. Leitura e escrita: uma abordagem cognitiva. Campinas: PSY II, 1994.

20. Denckla MB, Rudel RG. Rapid Automatized Naming of Pictured Objects, Colors, Letters and Numbers by Normal Children. Cortex. 1974; 10: 186-202.

21. Capellini SA, Tonelotto JMF, Ciasca SM. Medidas de desempenho escolar: avaliação formal e opinião de professores. Estud Psicol. 2004; 21( 2): 79-90.

22. Cavallini F, Berardo F, Perini S. Mental retardation and reading rate: effects of precision teaching. Life S Disabil. 2010; XIII (1): 87-101.

23. Chappell J, Stephens T, Kinnison L, Pettigrew $\mathrm{J}$. Educational diagnosticians' understanding of phonological awareness, phonemic awareness, and reading fluency. Assess Eff Interv. 2009; 35(1): 24-33.

24. Hayes R, Slater A. Three month-olds'detection of alliteration in syllables. Infant Behav Dev. 2008; (31): 153-6.

25. Manz P, Hughs C, Barnabas E, Bracaliello C. A desciriptive review and meta-analysis of familybased emergent literacy interventions: to what extent is the research applicable to low-income, ethnic-minority or linguistically-diverse young children? Early Child Res Q. 2010; 25: 409-31.

26. Ukrainetz T, Nuspl J, Wilkerson K, Beddes SR. The effects of syllable instruction on phonemic awareness in preschoolers. Early Child Res Q. 2010; 26(1), 50-60.

27. Archibald J. Phonologica features re-assembly and the importance of phonetic cues. Sec Lang Res. 2009; 25(2): 231-3.

28. Piasta SB, Wagner RK. Learning letter names and sounds: Effects of instruction, letter type, and phonological processing skill. J Exp Child Psychol. 2010; 105 (4): 324-44.

29. Gray A, McCutchen D. Young readers'use of phonological information: phonological awareness, memory, and comprehension. . J Learn Disabil. 2006; 39(4): 325-33.
30. Otaiba SA, Puranik C, Ziolkowski R, Montgomery T. Effectiveness of Early Phonological Awareness Interventions for Students With Speech or Language impairments. J Spec Educ. 2009; 43(2): 107-28.

31. Vaughn S, Cirino PT, Wanzek J, Wexler J, Fletcher JM, Dentón CD et al. Response to Intervention for Middle School Students With Reading Difficulties: Effects of a Primary and Secondary Intervention. School Psych Rev. 2010; 39(1): 3-21.

32. Ellis AW. Leitura, escrita e dislexia: uma análise cognitiva. Porto Alegre: Artes Médicas, 1995.

33. Torgensen JK, Wagner R, Rashotte CA, Burgess S Hetch S. Contributions of phonological awareness and rapid automatic naming ability to the growth of word-reading skills in second to fifth - grade children. Sci Stud Read. 1997; 1: 161-95.

34. Hawelka S, Gagl B, Wimmer H. A dual-route perspective on eye movements of dyslexic readers. Cognition. 2010; 115: 367-79.

35. Vukovic RK, Siegel LS. The double-deficit hypothesis: a comprehensive analysis of the evidence. J Learn Disabil. 2006; 39(1): 25-47.

36. Savage RS, Frederickson N, Goodwin R, Patni $\mathrm{U}$, Smith $\mathrm{N}$, Tuersley L. Relationships among rapid digit naming, phonological processing, motor automaticity, and speech perception in poor, average, an good readers and spellers. J Learn Disabil. 2005; 38(1): 12-28.

37. Villagrán MA, Guzmán JIN, Jiménez IM, Cuevas CA, Consejero EM, Olivier PR. Velocidad de nombrar y conciencia fonológica en el aprendizaje inicial de la lectura. Psicothema. 2010; 22(3): 436-42.

38. Frost SJ, Landi N, Mencl WE, Sandak R, Fulbright RK, Tejada ET, et al. Phonological awareness predicts activation patterns for print and speech. Ann Dyslexia (2009) 59:78-97.

39. Capellini SA, Lanza S. Desempenho de escolares em consciência fonológica, nomeação rápida, leitura e escrita. Pró-Fono. 2010; 22(3): 239-44.

http://dx.doi.org/10.1590/S1516-18462011005000140

RECEBIDO EM: 14/12/2010

ACEITO EM: 09/06/2011

Endereço para correspondência:

Giseli Donadon Germano

Rua Frei Jacinto, 264 - Fragata

Marília - SP

CEP: $17501-240$

E-mail: giseliger@yahoo.com.br 